\title{
Application Research on Fault Diagnosis of the Filter Unit Based on Intelligent Algorithm of GA and WNN
}

\author{
Zhao Xuejun $^{1,2}$, Wang Mingfang ${ }^{*}, 1$, Wang Jie $^{1}$, Tong Chuangming ${ }^{1}$ and Yuan Xiujiu ${ }^{2}$ \\ ${ }^{1}$ The College of Missile Air Force Engineering University, Xian, Shaanxi 710051, China \\ ${ }^{2}$ The College of Science Air Force Engineering University, Xian, Shaanxi 710051, China
}

\begin{abstract}
This paper focuses on the potential of GA algorithm for adaptive random global search, and WNN resolution as well as the ability of fault tolerance to build a multi intelligent algorithm based on the GA-WNN model using the filter unit of analog circuit for fault diagnosis. Construction of GA-WNN model was divided into two stages; in the first stage GA was used to optimize the initial weights, threshold, expansion factor and translation factor of WNN structure; while in the second stage, initially, based on WNN training and learning, global optimal solution was obtained. In the process of using analog output signal by using wavelet decomposition, the absolute value of coefficient of each frequency band sequence was obtained along with the energy characteristics of the cross joint, with a combination of feature vectors as the input of the neural network. Through the pretreatment method, in order to reduce the neural network input, neural grid size of neurons was reduced in each layer and the convergence speed of neural network was increased. The experimental results show that the method can diagnose single and multiple soft faults of the circuit, with high speed and high precision.
\end{abstract}

Keywords: Fault diagnosis, FFT, filter unit, GA, Intelligent algorithm, WNN.

\section{INTRODUCTION}

Fourier transform is a useful method for early signal analysis. Fourier transform is a global transformation of the signal in a time domain or frequency domain. However, in this kind of transformation, it is difficult to take care of the local nature, but some research work is needed to study local signals, such as non-stationary signal, which is difficult to analyze and research with Fourier transform. In order to solve this problem, researchers on one hand, improved the traditional Fourier transform, and on the other hand put forward some new methods of signal analysis, such as, short time Fourier transform and wavelet transform methods.

In the 1970s, analog circuit for fault diagnosis has been the hot topic of research in the field of circuit theory, and currently, it is still a very challenging leading problem. Its main difficulties involve three aspects: the first is the input and output in a continuous analog circuit; the second is analog circuits involving nonlinear problem; and the third is the discrete component parameters of analog circuit.

In the tentative study of analog circuit for fault diagnosis neural network is one of the many methods used in this process. For analyzing node voltage of the input data in neural network training, the trained neural network is used to find the faulty node which can effectively diagnose soft faults. *Address correspondence to this author at the The College of Missile Air
Force Engineering University, Xian, Shaanxi 710051, China;

E-mail: zywcczywec@163.com
The current research on soft fault diagnosis or single fault diagnosis [1-3], conforms to the fact that the fault diagnosis method is very rare. This paper proposed a method for fault diagnosis of circuit, to examine the sensitivity of the first analysis to determine the circuit nodes; used wavelet analysis to extract the characteristic signal used for training the neural network, and analyzed the performance of the network experiment which showed that this method is effective.

\section{THEWAVELETTRANSFORM}

Wavelet transform is a multi-resolution time-frequency signals analysis method, which can effectively represent the local characteristics of time and frequency domain. With low frequency and high frequency respectively and with temporal resolution and frequency resolution properties, it can analyze the abnormal transient signals accurately [4].

In comparison to fourier transform, wavelet transform has the following advantages:

1) With the resolution of the wavelet transform, window width (width) varies. Short window is used in high frequency, and the wide window is used in low frequency, which fully reflects the relative bandwidth frequency analysis and adaptive resolution analysis. It is consistent with time-varying signal characteristics. In order to analyze time-varying signals, it is very effective and is also called "mathematical microscope" [5].

2) Through spectrum analysis, wavelet transform is used to decompose signals that are of logarithmic form rather than a linear form. This approach can more effectively deal with time-varying signal [6]. 
3) In comparison, the wavelet transform and Fourier transform have uniformity and comparability as they are based on similar Planchera theorem and inverse formula, and their positive and inverse transform have the perfect symmetry, therefore, wavelet transform is considered to be a new form of Fourier transform.

4) The result of Fourier analysis shows that the theory is based on signal stability. For non-stationary signal, Fourier analysis may give false results, leading to faults in the diagnosis. For this kind of problem, wavelet analysis method has incomparable advantages [7]. Because of wavelet decomposition, especially in the wavelet packet decomposition technique, any signal (stationary or non-stationary) decomposes to the wavelet scale forms a cluster on the basic functions, yielding complete characterization of signal information channel sequence, along with the sequence of alternatives which can be used for in depth analysis, to obtain accurate fault diagnosis.

Dual wavelet is not necessarily based on one-to-one correspondence, however, it is preferred by researchers because dual wavelet with one-to-one correspondence has greater application value [8], which makes it a preferred model. The range of frequency in wavelet transform decreases with the increase in the decomposition scale and time range. But this cannot provide complete analysis, in a certain period of time, on all frequency domains or on all time domains within a certain band. In fact, there are a number of problems for certain period of time (or points) or frequency (or points) signal, thus, making the extraction of information of particular time and frequency difficult . Wavelet packet transform can solve such problems. With focus on frequency, it can improve the frequency resolution as well as the temporal resolution [9].

\subsection{Wavelet Transform Fault Feature Extraction}

By expanding the $\forall f(t) \in L^{2}(R)$ using wavelet function, the function becomes a continuous wavelet transform:

$W T_{f}(a, \tau)=\left\langle f(t), \Psi_{a, \tau}(t)\right\rangle=\frac{1}{\sqrt{a}} \int_{R} f(t) \Psi^{*}\left(\frac{t-\tau}{a}\right) d t$

The parameters $a$ and $\tau$ represent characterization of scale of wavelet transform through translation, and the function of time projection in the phase plane method promote the signal feature extraction.

Feature extraction is the key to determine a reasonable level of wavelet decomposition [10].Feature extraction layer of faults is more abundant, reducing the diagnosis speed, and is very important to achieve accurate balance. In this paper, Haar wavelet decomposition level had 5 layers.

\subsection{To Extract Fault Feature Analysis of Multi- Resolution}

Analog circuit fault usually belongs to the soft fault signal, with the change being small and suitable for describing high-frequency decomposition. Fault feature vectors were obtained by the following steps:
A) Signal decomposition, with $\mathrm{n}$ layer of Mallat decomposition algorithm results inn +1 coefficient $\left\{d_{1}, d_{2}, \cdots, d_{n+1}\right\}$;

B) Processing decomposition coefficient:

$D_{j}=\sum_{i=1}^{n+1}\left|d_{i}^{j}\right|$

C) Feature vector as $\left\{D_{n}, D_{n-1}, \cdots, D_{1}\right\}$, and the elements are placed in the order according to size;

D) Because of different characteristic parameters, it has great practical significance, which leads to inconsistent magnitude values, therefore, it must be normalized:

$D_{j}^{\prime}=\frac{D_{j}}{\sqrt{\sum_{j=1}^{n+1} D_{j}^{2}}}$

\section{THE GENETICALGORITHM}

GA (Genetic Algorithms) were invented by John Holland of University of Michigan, USA). and his students in the 1960s. It is a kind of biological evolution theory applied in computer system as an adaptive global optimization probability search algorithm. Classic genetic algorithm (GA) is the application of some fixed binary strings to the alternative solutions in encoding the fixed form of a individual binary string, with each having long binary strings in a population known as individuals, and Is any one of $\{0,1\}$.

Basic operators of genetic algorithm are: selection (Selection) operator, crossover (Crossover) operator and mutation operator [11] (Mutation):

The selection operator: It performs selection by calculating fitness value using the fitness function. Following this, by using a method of the past generation groups, individuals, who are absolutely fit, are transferred into the next generation population, on the basis of ideological source of survival of the fittest in nature.

(2) In the crossover operator: Crossover is another individual which matches random individuals in groups search, and on the basis of probability in part, it exchanges its chromosomes to generate new individuals. The crossover operation is the key of genetic algorithm.

(3) The mutation operator: Mutation is based on a random selection of certain individuals, according to the string data structure, with change in the probability of these individuals based on some string value. The probability of mutation is not high, but can meet the need of new individuals generated.

\section{INTELLIGENT ALGORITHM BASED ON GA AND WNN IN THE FILTER UNIT FAULT DIAGNOSIS APPLICATION}

Wavelets transform (WT) and neural network (NN) methods based on analog circuit for fault diagnosis were 
analyzed by classifying fault components corresponding to different faults. Wavelet analysis and neural network fault diagnosis were carried out based on following steps:

(1) The structure of neural network training samples and feature extraction Appropriate circuit node is selected as the test node. The node voltage analysis and feature extraction are carried out based on wavelet, and feature vector generating fault associated with all feature vectors.

(2) The design of neural network and training. By using the 3 layer BP network, node number $(\mathrm{N}+1) \mathrm{m}$; and number of hidden layer neurons $\sqrt{(N+1) m+k}+a$, are given as input, where $\mathrm{N}$ is the level of wavelet decomposition, $\mathrm{m}$ is the total number of test nodes, krepresents circuit elements for the state number, and ais an integer between 0-10. Neural network is used with LM (Levenberg-Marquardt) algorithm for training.

(3) The diagnosis of circuit fault. The neural network combined with feature vector is entered in the trained fault element, and the output corresponding to the element is diagnosed.

WNN (Wavelet Neural Network) method basically improves the fault tolerance ability and convergence speed of the Network parameters by adjusting relevance weights. It uses gradient descent method to optimize the network parameters, but restricts the parameter optimization, at the same time, the Network will easily fall into a local minimum and causes vibration. On the basis of the wavelet neural network method, genetic algorithm is introduced with natural selection and genetic principles.

Using random global search ability of GA, the WNN adaptive resolution and fault-tolerant ability, this paper used the GA - WNN model for research. The GA - WNN model is divided into two stages; The first stage of GA optimizes the initial weights of WNN structure, threshold value, scale factor and shift factor; in the second phase as in the previous phase based on WNN for training and learning, global optimal solution is obtained.

\subsection{Parameters of Wavelet Neural Network Structure Based on Genetic Algorithm}

Due to the random generation of initial weights in the neural network training process, the threshold expansion factor and translation parameters decrease the speed of network convergence. Thus, this ensures the origin of the network topology, using parameters optimization structure of wavelet neural network genetic algorithm.

1) According to the distribution weights randomly generate a set of chromosomes, including connection weights, threshold, scale and shift parameters for each layer of WNN. Then encode above parameters according to the coding method and initialize the population size, population number, genetic operator (crossover probability $P c$ and mutation probability $P m)$ and maximum number of generation.
2) Input training data into WNN and calculate error function value. Choose reciprocals of the sum of square of the error values as the individual fitness function values and sort the values.

3) Place the chromosome of the maximum fitness function value into next generation directly;

4) Perform crossover operation on Individual $G_{i}$ and $G_{i+1}$ with a crossover probalitity and obtain new individuals $G_{i}{ }^{\prime}$ and $G_{i+1}{ }^{\prime}$

5) Perform mutation operation on a individual with a mutation probility to generate a new individual;

6) Add the new individuals generated through above genetic operations into the population and calculate their fitness function values;

7) If the fitness function value meets the requirements, the network is continued to be built following the next step; otherwise, the above operation is repeated to optimize the network parameters, until the requirements are met;

8) Optimization result is decoded to obtain the parameters of wavelet neural network. The parameters can be used in the next phase of training neural network.

After completing the first stage, the structure parameters of WNN optimized by GA are chosen as the initial weights, threshold value, scale factor and shift parameter of network training so as to obtain their optimal values contributing to the further analog circuit fault diagnosis.

\subsection{Filter Unit Fault Diagnosis Example Based on GA and WNN}

Through a device filter element simulation experiment (Fig. 1), the effectiveness of the new fault diagnosis method was verified [9]. Experimental research was conducted on soft fault element, when the failure occurred in a component beyond the normal tolerance range. All capacitance tolerance was $10 \%$, and resistance tolerance was $5 \%$. Due to the sensitivity of fault diagnosis further analysis is needed to eliminate some of the parameters which are not effective for fault diagnosis. This section mainly diagnosed soft faults $\left(C_{1}, C_{2}, R_{2}, R_{3}\right)$ approx. eight, as well as diagnosed the normal mode. This section mainly diagnosed eight soft faults $\left(C_{1}, C_{2}, R_{2}, R_{3}\right)$ as well as a normal mode.

Circuit requires pulse excitation signal provided by the simulation software Pspice. The parameter values are shown in Table 1.

$\mathrm{V}_{0}$ denotes test point, and $R \uparrow, R \downarrow, C \uparrow, C \downarrow$ denotes the fault with $\uparrow$ representing too large and $\downarrow$ representing to small. The binary coding was used to express the status of these nine types of faults (including normal state), and related parameters are listed in Table 1. Each fault state performed 30 Monte Carlo analyses, underwent 20 neural network training, and examined 10 performances of the network. Decomposition of the circuit output signal and the resulting feature vector were given as input to the artificial 
neural network with wavelet. Using the neural network toolbox in Matlab construction of 3 layer BP network, the network training method for LM algorithm, the network parameters analyzed were: twelve input nodes; nine neurons in hidden layer, Tansig function as hidden layer transfer function ; four output neurons, purelin function as output transfer function; and the network training goal error being less than 0.01 . The experiment found that 66 trainings met the requirements. The error curve is shown in Fig. (2).

Table 1. Pulse excitation signal parameter value.

\begin{tabular}{|c|c|}
\hline Parameter Name & Parameter Value \\
\hline \hline Initial Value $\mathrm{V}_{1}$ & $1 \mathrm{mV}$ \\
\hline Ripple Value $\mathrm{V}_{2}$ & $5 \mathrm{mV}$ \\
\hline Initial Delay $\mathrm{T}_{\mathrm{D}}$ & $0.2 \mathrm{us}$ \\
\hline Rise Time $\mathrm{T}_{\mathrm{R}}$ & $0.1 \mathrm{us}$ \\
\hline Fall Time $\mathrm{T}_{\mathrm{F}}$ & 1 us \\
\hline Cycle $\mathrm{T}_{\mathrm{PER}}$ & 6 us \\
\hline Pulse Width $\mathrm{P}_{\mathrm{w}}$ & 3 us \\
\hline
\end{tabular}

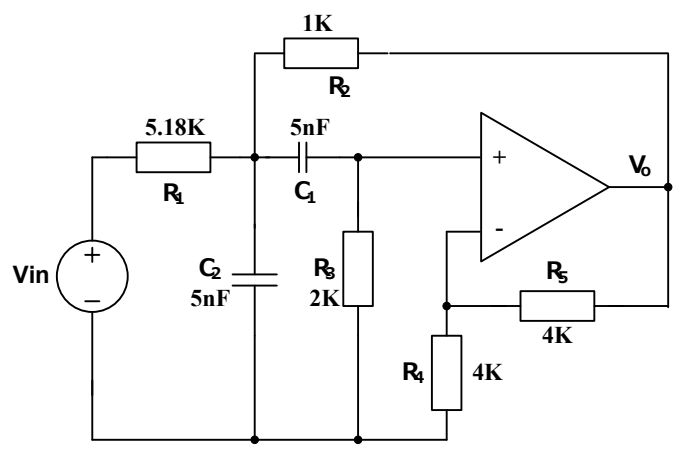

Fig. (1). The filter unit.

Table 2. Neural network to diagnose single soft fault results.

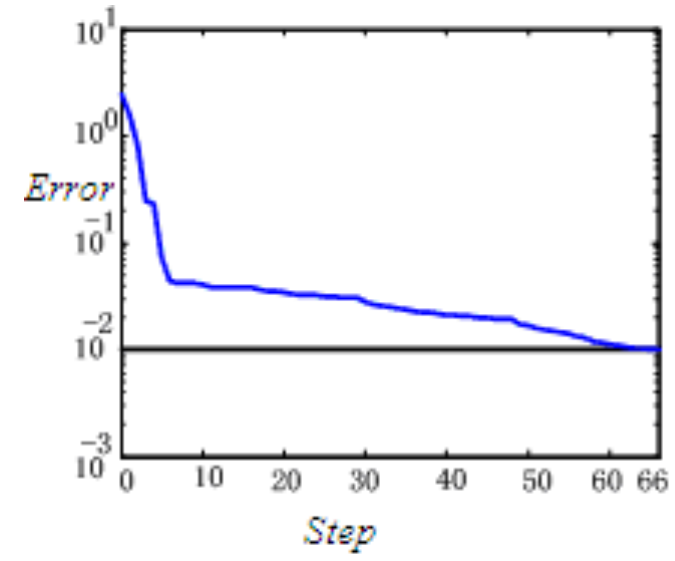

Fig. (2). Error curve.

By testing the single fault diagnosis performance of neural network, it was found that most of the network can accurately diagnose the fault, as the diagnostic rate was $97.18 \%$, which relates to the parameters shown in Table 2.

The same circuit simulation was used for multiple soft fault diagnosis. Due to the difference between multiple soft fault diagnosis and single soft fault diagnosis, between each fault state of the montecarlo analysis, the number increased to 80 times; 60 times for train network, 20 times for testing network.213 times after training error was less than 0.03 , and the accurate diagnostic rate of the neural network was 95.26\%.Diagnosis results are shown in Table 3 .

\section{CONCLUSION}

In this paper, wavelet neural network method was applied to fault diagnosis of analog circuits. By virtue of the wavelet decomposition of circuit output signal, the generated sum of the absolute value of coefficient sequence of each frequency band and energy features are crossed and combined, and the obtained feature vector is chosen as the input of neural network. The pretreatment method was used by reducing the input of neural network, to reduce the grid size of neurons in each layer, and increase the convergence speed of neural

\begin{tabular}{|c|c|c|c|c|c|c|c|c|}
\hline \multirow{2}{*}{$\begin{array}{c}\text { Nominal } \\
\text { Valve }\end{array}$} & \multirow{2}{*}{$\begin{array}{l}\text { Failure } \\
\text { Mode }\end{array}$} & \multirow{2}{*}{$\begin{array}{c}\text { Failure } \\
\text { value }\end{array}$} & \multicolumn{4}{|c|}{ Ouput of Neural Network } & \multirow{2}{*}{$\begin{array}{c}\text { Approximate } \\
\text { Binary Code } \\
\text { Actual } \\
\text { Output }\end{array}$} & \multirow{2}{*}{$\begin{array}{c}\text { Approximate } \\
\text { Binary Code } \\
\text { Expected } \\
\text { Output }\end{array}$} \\
\hline & & & Output 1 & Output 2 & Ovtpux 3 & Ovtput 4 & & \\
\hline & Normal & & -0.00286 & 0.002242 & 0.081705 & -0.02923 & 0000 & 0000 \\
\hline $5 \mathrm{nF}$ & Cl large & $8 \mathrm{nF}$ & 0.008056 & 0.436053 & 0.445316 & 0.967664 & 0001 & 0001 \\
\hline $5 \mathrm{nF}$ & $\mathrm{Cl}$ small & $2 \mathrm{nF}$ & -0.00298 & 0.001489 & 1.015543 & 0.013681 & 0010 & 0010 \\
\hline $5 \mathrm{nF}$ & C2 large & $8 \mathrm{nF}$ & 0.005906 & 0.1171 & 1.047508 & 1.294471 & 0011 & 0011 \\
\hline $5 \mathrm{nF}$ & C2 large & $2 \mathrm{nF}$ & 0.00209 & 0.982804 & -0.02105 & -0.02577 & 0100 & 0100 \\
\hline $1 \mathrm{~K}$ & R.2 large & $3 \mathrm{~K}$ & -0.04361 & 1.074272 & -0.09839 & 0.865281 & 0101 & 0101 \\
\hline $1 \mathrm{~K}$ & R2 small & $0.4 \mathrm{~K}$ & -0.00391 & 0.904425 & 0.827933 & 0.04256 & 0110 & 0110 \\
\hline $2 \mathrm{~K}$ & R.3 large & $5 \mathrm{~K}$ & -0.0035 & 1.003027 & 1.138703 & 0.938824 & 0111 & 0111 \\
\hline $2 \mathrm{~K}$ & R3 small & $0.8 \mathrm{~K}$ & 0.957869 & -0.00199 & 0.017849 & 0.002452 & 1000 & 1000 \\
\hline
\end{tabular}


Table 3. Neural network diagnosis of multiple soft fault results.

\begin{tabular}{|c|c|c|c|c|c|c|}
\hline \multirow{2}{*}{ Failure Mode } & \multicolumn{4}{|c|}{ Output of Neural Network } & \multirow{2}{*}{$\begin{array}{l}\text { Approximate } \\
\text { Binary Code } \\
\text { Actual Output }\end{array}$} & \multirow{2}{*}{$\begin{array}{l}\text { Approximate } \\
\text { Binary Code } \\
\text { Expected Output }\end{array}$} \\
\hline & Output 1 & Output 2 & Output 3 & Output 4 & & \\
\hline Normal & 0.26475 & 0.003513 & 0.000412 & 0.249928 & 0000 & 0000 \\
\hline $\mathrm{C}_{1} \uparrow \mathrm{C}_{2 \downarrow} \downarrow$ & 0.059627 & -0.01038 & -0.00399 & 1.070899 & 0001 & 0001 \\
\hline $\mathrm{C}_{1} \downarrow \mathrm{R}_{2 \downarrow} \downarrow$ & -0.00337 & 0.007431 & 0.913782 & 0.010877 & 0010 & 0010 \\
\hline $\mathrm{C} 2 \uparrow \mathrm{R} 3 \uparrow \uparrow$ & -0.00489 & 0.009979 & 0.984525 & 0.996891 & 0011 & 0011 \\
\hline $\mathrm{R}_{2} \uparrow \mathrm{R}_{3} \downarrow \uparrow$ & -0.01194 & 0.990586 & -0.01203 & 0.02165 & 0100 & 0100 \\
\hline $\mathrm{C}_{1} \downarrow \mathrm{C}_{2} \uparrow \mathrm{R}_{2} \uparrow \uparrow$ & -0.00305 & 0.936965 & -0.00269 & 0.99755 & 0101 & 0101 \\
\hline $\mathrm{C}_{1} \uparrow \mathrm{C}_{2} \downarrow \mathrm{R}_{3} \downarrow \downarrow$ & -0.00223 & 1.093526 & 1.064728 & -0.00127 & 0110 & 0110 \\
\hline $\mathrm{C}_{1} \downarrow \mathrm{R}_{2} \uparrow \mathrm{R}_{3} \uparrow \uparrow$ & -0.02158 & 1.060578 & 0.903843 & 1.039209 & 0111 & 0111 \\
\hline $\mathrm{C}_{2} \uparrow \mathrm{R}_{2} \downarrow \mathrm{R}_{3} \downarrow \uparrow$ & 0.963493 & 0.024187 & 0.026147 & -0.00099 & 1000 & 1000 \\
\hline $\mathrm{C}_{1} \uparrow \mathrm{C}_{2} \uparrow \mathrm{R}_{2} \uparrow \mathrm{R}_{3} \uparrow \uparrow$ & 1.016961 & -0.00956 & -0.02835 & 0.934815 & 1001 & 1001 \\
\hline
\end{tabular}

network effectively. The experimental results show that the new method can diagnose circuit of single soft faults and multiple soft fault effectively, and its diagnostic rate is fast and has high diagnostic accuracy .

\section{CONFLICT OF INTEREST}

The authors confirm that this article content has no conflict of interest.

\section{ACKNOWLEDGEMENTS}

This work was supported by Aeronautical Science Fundof China No.20120196006and Electronic Information in Shaanxi Province Key Laboratory of System Integration Fund No.201113y14.

\section{REFERENCES}

[1] J. Wu, J. Zhu, G. Song, B. Ni, "Missile support equipment development to explore the, Cruise Missile, vol. 09, pp. 39-42, 2004.

[2] W. Wang, "Military technology cooperation of China Russia strategic cooperative partnership under the Russian study," Research on Russian Central Asian and Eastern Europe, vol. 04, pp. 62-68, 2006
[3] W. Lv, X. Li, and D. Wang, "Standardize the order of strengthening equipment management problem research journal", Equipment Institute, vol. 2, pp.10-13, 2014.

[4] M. Elsayed, "An overview of wavelet analysis and its application to ocean wind waves," Journal of Coastal Research, vol. 263, 2010.

[5] M.Yu, "SecondWavelet Transform in the Fault Diagnosis of Rotating Machinery Application Research," Nanjing University of Aeronautics, China, 2009.

[6] D.You, "The Intelligent Diagnosis Technology and Implementation of Fault of Reciprocating Pump Valve," China: Daqing Petroleum Institute, 2004.

[7] M. A. K. Elsayed, "Application of continuous wavelet analysis in distinguishing breaking and nonbreaking waves in the wind-wave time series," Journal of Coastal Research, vol. 24, no.1, pp. 273277, 2008.

[8] M. S. Naderia, T.R. Blackburna, B.T. Phunga, Mehdi S. Naderia, A. Nasiri, "Application of wavelet analysis to the determination of partial discharge location in multiple- transformer windings," Electric Power Systems Research Alpha, vol. 78, no. 2, 2007.

[9] Y. Sima, "Damage detection in structures using modified backpropagation neural networks," Acta. Mechanica. Solida. Sinica, vol. 04 , pp. 358-370, 2002

[10] M. Górski, M. Marcinek,"Application of selected software tools for data collection and analysis in library management and their effectiveness assessment: results of the research conducted at Polish Academic Libraries", In: Integrated and Strategic Advancements in Decision Making Support Systems 2012, pp. 108-119.

[11] W. Liu, "Genetic Algorithm and its Application in Constrained Optimization”, China: Tianjin University, 2012. 